\title{
Combine Received Signal Strength and Angle of Arrival based Localization Technique for WSN
}

\author{
Kapil Uraiya \\ Research Scholar (EC), TIT, \\ Bhopal, India
}

\author{
Dilip Kumar Gandhi \\ Department of EC, TIT, \\ Bhopal, India
}

\begin{abstract}
In wireless sensor network nodes position estimation in space is known as localization. It plays a critical role in many locations critical applications (like target tracking) where inaccurate location information, can effectively degrade the service performance.The most reliable technique for the localization is GPS but the cost and power requirement makes it's unfeasible for most of the applications. In search of a substitute for GPS, landmark or anchor node (which have perfect information about their positions or coordinates) is used to transmit some reference signal and the other nodes are used some algorithms (trilateration or triangulation etc.) to estimate their position on the basis of reference signal strength (used for distance estimation) and direction(used for angle estimation).However the estimation of distance from signal strength decay in not very accurate especially in time varying environmental conditions and the estimation of exact direction required highly directive antenna but, may still affected by multipath fading. Hence in this paper we are presenting a combine distance and angle based optimization approach which tries to find the optimal location by satisfying both the criteria with minimal error. The simulation results also validates that the proposed algorithm effectively outperform both the techniques.
\end{abstract}

Keywords: WSN, Localization, Optimization, Genetic Algorithm (GA).

\section{INTRODUCTION}

WSN is a specially designed data communication system which does not requires any centralized control or infrastructure for application areas where the establishments of such resources are difficult like military, industrial, marine and in natural disasters monitoring, early warning, rescuing and other emergency situations, recently its area of application is also growing in household and medical fields. The requirement and importance of localization for WSN could be understood by its application in atmospheric or geological monitoring where the sensor reading are very specific to height on atmosphere or the depth of sensor underground. Since the estimation of distance on the basis of received signal strength (RSS) in not very accurate because of the fading characteristics of the path greatly varies with time and weather also the angle of arrival (AOA) estimation either required a highly directional antenna or array antenna structure with complex processing algorithm but still error cannot be neglected. The other problem is with localization is it required higher numbers of anchor points to exactly estimate the position in three dimensional space of node. The methods discusses above are fall in the range based techniques however there exist another approach which uses only the connectivity information between unknown nodes and landmarks. These techniques can be further divided into two categories: local techniques and hop counting technique. In hop counting technique node estimates the distances to its neighbor anchor nodesby the hop counts and the hop size for the closest anchor node and then estimate its own position, while the local technique node collects the position information of its neighboranchor nodes to estimate its position. In this paper we are focusing on the range based technique because of its accuracy and adaptability to any protocol and presented a combine RSS and AOA based optimization approach to accurately estimate the location of node. This rest of paper is organized as follow,section II presents a brief review of the related literature while the III and IV section explains the RSS and AOA techniques respectively for location estimation. The section $\mathrm{V}$ explains the genetic algorithm and section VI explains the proposed algorithm follower by the simulation results and conclusion with future scope in VII and VIII respectively.

\section{LITERATURE REVIEW}

A survey on different localization techniques available is presented by Guangjie Han et al [1], they also reclassify thelocalization algorithms on the mobility state of landmarks and unknown nodes point of viewwith a detailed analysis.Distributed Angle Estimation based approach is presented in [2]. In the literature two antenna anchors are used totransmit linear chirp wavessimultaneously, and the angle of departure (AOD) of the emitted waves at each receiving node is estimated via frequencymeasurement of the local received signal strength indication (RSSI) signal [3]. Estimation method is also improved with the adaption of multiple parallel arrays to providethe space diversity. The other advantage of the technique is rely only on radio transceivers and synchronization is needed. Zero-configuration indoor localization to estimate relationships between RSSI samples and the distance between nodes is presented in [4]. A localization approach specifically for the mine environments proposed in [5]. They proposed an automatic approach for simultaneous refinement ofsensors positions and target tracking by the application of ameasurement model from a real mine, and apply a discretevariant of real-time belief propagationto handle all non-Gaussianuncertainties typical for mining environments. Mohammad Abdul Azim et al [6] presented a cross entropy (CE) method for localization of nodes. Their proposedcentralized algorithm estimates location of the nodes by measuring distances of the neighboring nodes [7]. Finally the error minimization is done by using the CEmethod. The sensor localization for the situations where the anchor power is unknown is proposed in [8] which utilizes the semi-definite programming (SDP) relaxation technique and the algorithm does not requires anchor power information it requires only an estimate of the path loss exponent (PLE) [9] [10]. 


\section{LOCALIZATION ALGORITH}

\subsection{Received Signal Strength Based}

\section{Localization}

By definition, the received signal strength is the voltage or power measured at the receiver end using signal strength indicator (RSSI) circuit. Since signal strength estimator is presently comes as an integral part of radio receiver chiphence does not requires hardware components. Since only signal strength is needed the technique does not imposed additional network traffic overhead.

Following derivation are used for finding distance from signal strength:

Let the transmission power of anchor node $=P_{t x}$

The strength estimated at receiver node $=P_{r x}$

Assuming that path - loss model is known

The path - loss coefficient $=\alpha$

Then the following equation can be used for estimation of distance between anchor node and the receiver nodes:

$$
\begin{array}{r}
P_{r x}=c * \frac{P_{t x}}{d^{\alpha}} \\
d=\sqrt[\alpha]{c * \frac{P_{t x}}{P_{r x}} \ldots \ldots \ldots . .}
\end{array}
$$

\section{Where}

$c=$ constant dependent on the path - loss.

$\alpha=2$, since received power is inversaly proportonal to

distance.

$2 \leq \alpha \leq 4=$ for the multipath fading channel and

spreadi spectrum transmistion technique .

Ones the node estimates the distance from different anchor nodes it utilizes the following algorithm to estimate its location

Let the total number of anchor nodes $=n$

let the coordinates of these nodes $=\left(x_{i}, y_{i}, z_{i}\right), i \in n$

let the coordinates of the node to be estimated

$$
=\left(x_{u}, y_{u}, z_{u}\right)
$$

Estimated distances from each anchor node using RSS

$$
=d_{i, e s t}, i \in n
$$

Writing the equalities,

$$
\sqrt{\left(x_{i}-x_{u}\right)^{2}+\left(y_{i}-y_{u}\right)^{2}+\left(z_{i}-z_{u}\right)^{2}}=d_{i, e s t}
$$

for each $i \in n$

$$
\begin{gathered}
o b j_{f u n}=\sum_{i=1}^{n} \mid \sqrt{\left(x_{i}-x_{u}\right)^{2}+\left(y_{i}-y_{u}\right)^{2}+\left(z_{i}-z_{u}\right)^{2}} \\
-d_{i, e s t} \mid
\end{gathered}
$$

Hence the location of node can be estimated by searching the values of $\left(x_{u}, y_{u}, z_{u}\right)$ which satisfies the equation (2) or minimizing the value of objective function (equation (3)).

\subsection{Angle-of-Arrival based localization}

The angle-of-arrival (AOA) is the angle of the receiving signal respective to receiver's position.

Presently two different techniques are used for the estimation of AOA.

In the first technique receiver utilizes the array antenna stricture and the received signal from each elements of the array is then processed to estimate the AOA utilizing the array antenna properties.

The second technique for measuring the source signal's AOA, utilizes the rotating, directional antennas, and the angle is estimated by observing the peaks. The rotational angle between two peaks represents the relative angle between for the receiver's point of view.

The relation between relative angles and the coordinates is given as follows

$$
\theta=2 * \operatorname{atan}\left(\frac{\operatorname{norm}\left(v_{u} * \operatorname{norm}\left(v_{i}\right)-\operatorname{norm}\left(v_{u}\right) * v_{i}\right)}{\operatorname{norm}\left(v_{u} * \operatorname{norm}\left(v_{i}\right)+\operatorname{norm}\left(v_{u}\right) * v_{i}\right)}\right)
$$

Now ones the angle is estimated from all anchor points the location vector can be calculated by minimizing the equation (5)

$$
\begin{aligned}
& \sum_{i=1}^{o b j_{\text {fun }}} \mid 2 \\
& * \operatorname{atan}\left(\frac{\operatorname{norm}\left(v_{u} * \operatorname{norm}\left(v_{i}\right)-\operatorname{norm}\left(v_{u}\right) * v_{i}\right)}{\operatorname{norm}\left(v_{u} * \operatorname{norm}\left(v_{i}\right)+\operatorname{norm}\left(v_{u}\right) * v_{i}\right)}\right)-\theta_{i, \text { est }} \mid
\end{aligned}
$$

where $\theta_{i, e s t}=$ estimated angles with the $\mathrm{i}^{\mathrm{th}}$ anchor node.

\section{GENETIC ALGORITHM}

A simple Genetic Algorithm is an iterative procedure, which maintains a constant size population $\mathrm{P}$ of candidate solutions. During each iteration step (generation) three genetic operators (reproduction, crossover, and mutation) are performing to generate new populations (offspring), and the chromosomes of the new populations are evaluated via the value of the fitness which is related to cost function. Based on these genetic operators and the evaluations, the better new populations of candidate solution are formed. With the above description, a simple genetic algorithm is given as follow: 
1. Generate randomly a population of binary string

2. Calculate the fitness for each string in the population

3. Create offspring strings through reproduction, crossover and mutation operation.

4. Evaluate the new strings and calculate the fitness for each string (chromosome).

5. If the search goal is achieved, or an allowable generation is attained, return the best chromosome as the solution; otherwise go to step 3 .

\section{PROPOSED WORK}

The proposed system estimates the optimal location of node from the available anchor nodes by using RSS and AOA and finding the optimal solution for both simultaneously. The proposed algorithm can be described in following steps

Step 1: let in the present topology of the network $\mathrm{N}$-anchor nodes with their known location are present and all of them are transmitting their locations and the power and if they are not transmitting these information it is assumed that the nodes already have these information.

Step 2: know the node wants to locate estimates the signal strength of the signal received form each anchor nodes separately and uses the equation (1) to estimate the approximate distance from each of the anchor nodes.

Step 3: Ones the node estimates the distance from all the anchor nodes it starts finding the angle of arrival from each nodes by either using array antenna processing or by simple directional rotating antenna.

Step 4: After calculating the information of distance and angles the node uses the genetic algorithm to find its coordinates such that in minimizes the objective function given in equation (7)

$$
\begin{aligned}
& o b j_{f u n} \\
& =\sum_{i=1}^{n} \mid \sqrt{\left(x_{i}-x_{u}\right)^{2}+\left(y_{i}-y_{u}\right)^{2}+\left(z_{i}-z_{u}\right)^{2}} \\
& -d_{i, e s t} \\
& +\mid 2 \\
& \text { * } \operatorname{atan}\left(\frac{\operatorname{norm}\left(v_{u} * \operatorname{norm}\left(v_{i}\right)-\operatorname{norm}\left(v_{u}\right) * v_{i}\right)}{\operatorname{norm}\left(v_{u} * \operatorname{norm}\left(v_{i}\right)+\operatorname{norm}\left(v_{u}\right) * v_{i}\right)}\right) \\
& -\theta_{i, e s t} \mid \text {. }
\end{aligned}
$$

Step 5: if the genetic algorithm finds a solution for the equation 8 it terminates and the returns the solution otherwise it gives the best fitted solution achieved within the given iterations.

\section{SIMULATION RESULTS}

The evaluation of the proposed work is done by simulating it for different scenarios and configurations.

\section{Scenario 1:}

Table 1: Configuration used for scenario 1 to evaluation of the proposed algorithm.

\begin{tabular}{|c|c|}
\hline Properties & Value \\
\hline Width & $100 \mathrm{~m}$ \\
\hline Height & $100 \mathrm{~m}$ \\
\hline Length & $100 \mathrm{~m}$ \\
\hline Number of Anchor Nodes & 2 \\
\hline Error in Distance Calc. (\%) & 5 \\
\hline Error in Angle Calc. (\%) & 5 \\
\hline GA Population Size & 64 \\
\hline Maximum Iterations & 100 \\
\hline
\end{tabular}

\begin{tabular}{|c|c|c|c|c|c|}
\hline Technique & $\mathrm{x}$ & $\mathrm{y}$ & $\mathrm{z}$ & $\%$ Error & $\begin{array}{c}\text { Time } \\
(\text { Sec. })\end{array}$ \\
\hline Original & 36.9847 & 4.8727 & 65.6513 & 0 & 0 \\
\hline RSS & 14.4015 & 20.4762 & 76.325 & 29.4516 & 38.178 \\
\hline AOA & 66.3084 & 1.8406 & 61.9508 & 29.7114 & 36.7052 \\
\hline Proposed & 36.0691 & 15.7879 & 81.3745 & 19.1624 & 54.2971 \\
\hline
\end{tabular}

Scenario 2:

Table 2: Configuration used for scenario 2 to evaluation of the proposed algorithm.

\begin{tabular}{|c|c|}
\hline Properties & Value \\
\hline Width & $100 \mathrm{~m}$ \\
\hline Height & $100 \mathrm{~m}$ \\
\hline Length & $100 \mathrm{~m}$ \\
\hline Number of Anchor Nodes & 3 \\
\hline Error in Distance Calc. (\%) & 5 \\
\hline Error in Angle Calc. (\%) & 5 \\
\hline GA Population Size & 64 \\
\hline Maximum Iterations & 100 \\
\hline
\end{tabular}

\begin{tabular}{|c|c|c|c|c|c|}
\hline $\begin{array}{c}\text { Techniqu } \\
\mathrm{e}\end{array}$ & $\mathrm{x}$ & $\mathrm{y}$ & $\mathrm{z}$ & $\begin{array}{c}\% \\
\text { Error }\end{array}$ & $\begin{array}{c}\text { Time } \\
(\text { Sec. })\end{array}$ \\
\hline Original & $\begin{array}{c}61.954 \\
8\end{array}$ & $\begin{array}{c}29.690 \\
1\end{array}$ & $\begin{array}{c}33.572 \\
4\end{array}$ & 0 & 0 \\
\hline RSS & $\begin{array}{c}57.439 \\
9\end{array}$ & $\begin{array}{c}31.209 \\
9\end{array}$ & $\begin{array}{c}34.075 \\
5\end{array}$ & $\begin{array}{c}4.790 \\
3\end{array}$ & $\begin{array}{c}28.144 \\
6\end{array}$ \\
\hline AOA & 61.205 & $\begin{array}{c}28.567 \\
8\end{array}$ & 33.239 & $\begin{array}{c}1.390 \\
3\end{array}$ & $\begin{array}{c}28.494 \\
5\end{array}$ \\
\hline Proposed & $\begin{array}{c}61.527 \\
4\end{array}$ & $\begin{array}{c}29.007 \\
1\end{array}$ & $\begin{array}{c}33.787 \\
5\end{array}$ & 1.03 & $\begin{array}{c}44.672 \\
8\end{array}$ \\
\hline
\end{tabular}

\section{Scenario 3:}

Table 3: Configuration used for scenario 3 to evaluation of the proposed algorithm.

\begin{tabular}{|c|c|}
\hline Properties & Value \\
\hline Width & $100 \mathrm{~m}$ \\
\hline Height & $100 \mathrm{~m}$ \\
\hline Length & $100 \mathrm{~m}$ \\
\hline Number of Anchor Nodes & 4 \\
\hline Error in Distance Calc. (\%) & 5 \\
\hline Error in Angle Calc. (\%) & 5 \\
\hline GA Population Size & 64 \\
\hline Maximum Iterations & 100 \\
\hline
\end{tabular}




\begin{tabular}{|c|c|c|c|c|c|}
\hline $\begin{array}{c}\text { Techniqu } \\
\mathrm{e}\end{array}$ & $\mathrm{x}$ & $\mathrm{y}$ & $\mathrm{z}$ & $\begin{array}{c}\% \\
\text { Error }\end{array}$ & $\begin{array}{c}\text { Time } \\
(\mathrm{Sec} .)\end{array}$ \\
\hline Original & $\begin{array}{c}49.091 \\
9\end{array}$ & $\begin{array}{c}42.383 \\
1\end{array}$ & $\begin{array}{c}64.113 \\
4\end{array}$ & 0 & 0 \\
\hline RSS & $\begin{array}{c}45.885 \\
6\end{array}$ & $\begin{array}{c}44.471 \\
4\end{array}$ & $\begin{array}{c}65.907 \\
9\end{array}$ & $\begin{array}{c}4.226 \\
3\end{array}$ & $\begin{array}{c}37.629 \\
2\end{array}$ \\
\hline \multirow{2}{*}{ AOA } & 52.692 & $\begin{array}{c}41.801 \\
3\end{array}$ & $\begin{array}{c}64.185 \\
6\end{array}$ & $\begin{array}{c}3.647 \\
6\end{array}$ & $\begin{array}{c}37.342 \\
1\end{array}$ \\
\hline Proposed & $\begin{array}{c}50.070 \\
3\end{array}$ & $\begin{array}{c}44.080 \\
1\end{array}$ & $\begin{array}{c}64.896 \\
8\end{array}$ & $\begin{array}{c}2.109 \\
7\end{array}$ & $\begin{array}{c}57.985 \\
3\end{array}$ \\
\hline
\end{tabular}

\section{Scenario 4:}

Table 4: Configuration used for scenario 1 to evaluation of the proposed algorithm.

\begin{tabular}{|c|c|}
\hline Properties & Value \\
\hline Width & $100 \mathrm{~m}$ \\
\hline Height & $100 \mathrm{~m}$ \\
\hline Length & $100 \mathrm{~m}$ \\
\hline Number of Anchor Nodes & 5 \\
\hline Error in Distance Calc. (\%) & 5 \\
\hline Error in Angle Calc. (\%) & 5 \\
\hline GA Population Size & 64 \\
\hline Maximum Iterations & 100 \\
\hline
\end{tabular}

\begin{tabular}{|c|c|c|c|c|c|}
\hline $\begin{array}{c}\text { Techniqu } \\
\mathrm{e}\end{array}$ & $\mathrm{x}$ & $\mathrm{y}$ & $\mathrm{z}$ & $\begin{array}{c}\% \\
\text { Error }\end{array}$ & $\begin{array}{c}\text { Time } \\
(\mathrm{Sec} .)\end{array}$ \\
\hline Original & $\begin{array}{c}26.897 \\
4\end{array}$ & $\begin{array}{c}49.514 \\
4\end{array}$ & $\begin{array}{c}43.770 \\
1\end{array}$ & 0 & 0 \\
\hline \multirow{2}{*}{ RSS } & $\begin{array}{c}27.018 \\
4\end{array}$ & $\begin{array}{c}48.221 \\
1\end{array}$ & $\begin{array}{c}41.680 \\
2\end{array}$ & $\begin{array}{c}2.460 \\
6\end{array}$ & $\begin{array}{c}39.276 \\
9\end{array}$ \\
\hline \multirow{2}{*}{$\mathrm{AOA}$} & $\begin{array}{c}27.480 \\
4\end{array}$ & $\begin{array}{c}49.563 \\
4\end{array}$ & $\begin{array}{c}46.146 \\
6\end{array}$ & $\begin{array}{c}2.447 \\
5\end{array}$ & $\begin{array}{c}39.457 \\
1\end{array}$ \\
\hline \multirow{2}{*}{ Proposed } & $\begin{array}{c}25.719 \\
3\end{array}$ & $\begin{array}{c}49.288 \\
5\end{array}$ & $\begin{array}{c}45.754 \\
3\end{array}$ & $\begin{array}{c}2.318 \\
6\end{array}$ & $\begin{array}{c}56.535 \\
6\end{array}$ \\
\hline
\end{tabular}

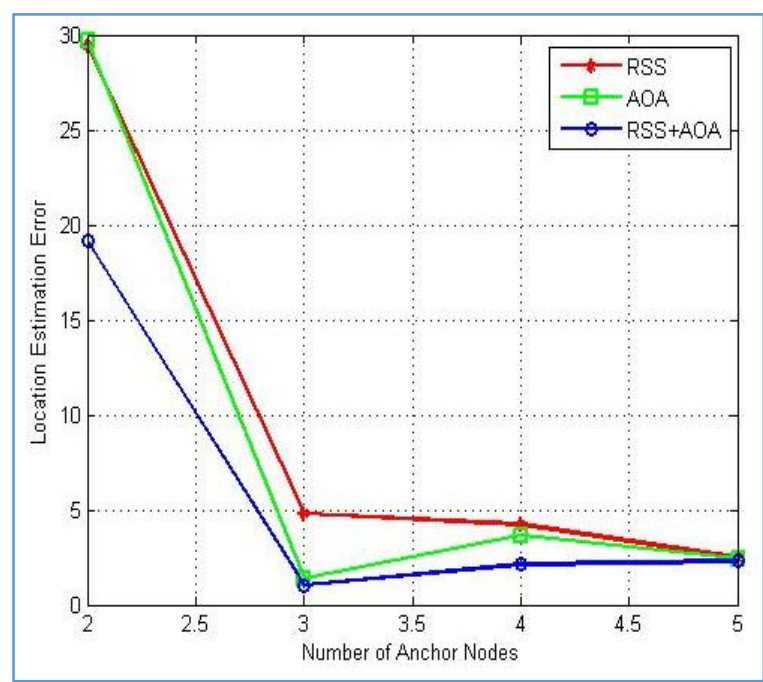

Figure 1: Comparison of the proposed algorithm (RSS+AOA) with RSS and AOA for the Location Estimation Error (in Percentage).

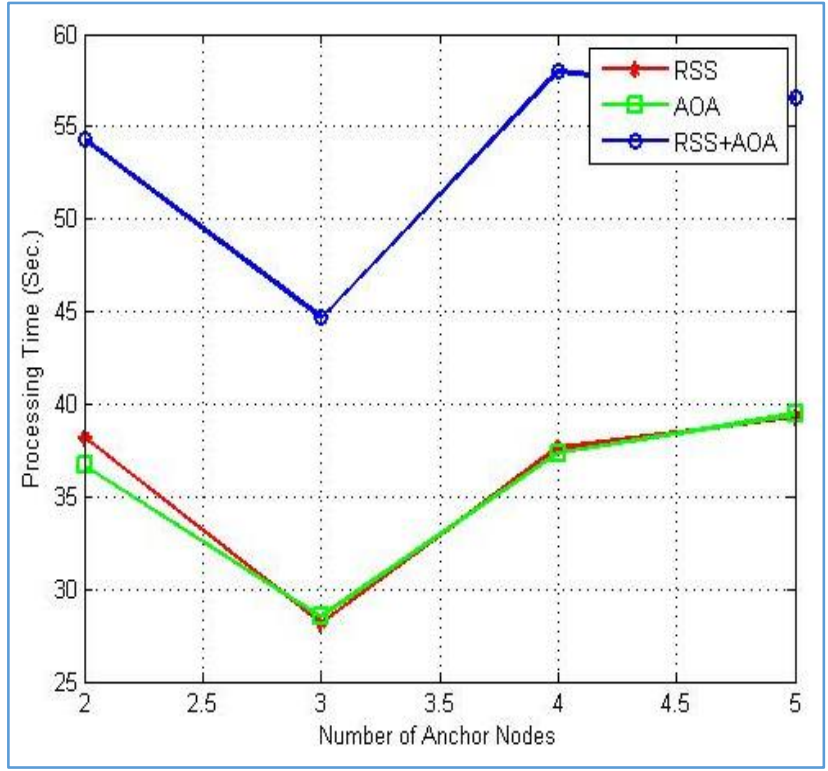

Figure 2: Comparison of the proposed algorithm (RSS+AOA) with RSS and AOA for the Location Estimation Time (in Seconds).

\section{CONCLUSION AND FUTURE ASPECTS}

This paper proposes a combine RSS + AOA based algorithm which are simultaneously optimized by the genetic algorithm to find the optimal solution of the location of the sensor node using some anchor nodes. The simulation results with different scenario shows that the present algorithm gives the highest accuracy with a minimum error of $1 \%$ with is twice better than the closest competitor AOA. The result also indicates that only three anchor node are sufficient to provide best estimation the further increase in anchor node leads to increase in time but does not improves accuracy. The present simulation also shows that the processing time for the proposed algorithm is much higher than others this is because standard genetic algorithm is used however in future some dedicated optimization technique can be developed but presently it is leave for future work.

\section{REFERENCES}

[1] Guangjie Han, Huihui Xu, Trung Q. Duong, Jinfang Jiang and Takahiro Hara" Localization algorithms of Wireless Sensor Networks: a survey", Telecommunication Systems Modeling, Analysis, Design and Management ISSN 1018-Telecommun System, DOI 10.1007/s11235-011-9564-7.

[2] Weile Zhang, Qinye Yin, Hongyang Chen, Feifei Gao, Member, and Nirwan Ansari "Distributed Angle Estimation for Localization inWireless Sensor Networks", IEEE TRANSACTIONS ON WIRELESS COMMUNICATIONS, VOL. 12, NO. 2, FEBRUARY 2013.

[3] Alessandro Redondi, Marco Chirico, Luca Borsani, Matteo Cesana, Marco Tagliasacchi "An integrated system based on wireless sensor networks for patientmonitoring, localization and tracking", Ad Hoc Networks 11 (2013) 39-53. 
[4] Mohammad Abdul Azim, Zeyar Aung, Weidong Michael Xiao and VinodKhadkikar "Localization in Wireless Sensor Networks by CrossEntropy Method", Data \& Network Analytics Research Group (DNA).

[5] Chenshu Wu, Zheng Yang, Yunhao Liu, and Wei Xi, "WILL: Wireless Indoor Localization without Site Survey" IEEE Transactions On Parallel And Distributed Systems, Vol. 24, No. 4, April 2013.

[6] Zheng Yang, Chenshu Wu, Tao Chen, , ,Yiyang Zhao, Wei Gong, and Yunhao Liu, "Detecting Outlier Measurements Based on Graph Rigidity for Wireless Sensor Network Localization" IEEE Transactions On Vehicular Technology, Vol. 62, No. 1, January 2013.

[7] Jianbin Fu And Shilong Pan, "Uwb-Over-Fiber Sensor Network For Accurate Localization Based On Optical Time-Division Multiplexing” pans@ieee.org
[8] Shun Kawakami and Tomoaki Ohtsuki , "Localization using iterative angle of arrival method sharing snapshots of coherent subarrays" Kawakami and Ohtsuki EURASIP Journal on Advances in Signal Processing2011,2011:46http://asp.eurasipjournals.com/c ontent/2011/1/46

[9] Reza Monir Vaghefi and Mohammad Reza Gholami "Cooperative Received Signal Strength-Based Sensor Localization with Unknown Transmit" Copyright (c) 2012 IEEE.

[10] Chi-Chang Chen, Chi-Yu Chang, and Yan-Nong Li "Range-Free Localization Scheme in Wireless Sensor Networks Based on Bilateration" Copyright () 2013 ChiChang Chen et al.

[11] Xinyu WANG, and Ziwen SUN "Genetic Algorithm for Wireless Sensor Network Localization With Level-based Reliability Scheme” 1553-9105 / Copyright @ 2013 